\title{
Photon-Induced Near Field Electron Microscopy
}

\author{
Sang Tae Park and Ahmed H. Zewail \\ Physical Biology Center for Ultrafast Science and Technology, Arthur Amos Noyes Laboratory \\ of Chemical Physics, California Institute of Technology, Pasadena, California 91125, U.S.A.
}

\begin{abstract}
Ultrafast electron microscopy in the space and time domains utilizes a pulsed electron probe to directly map structural dynamics of nanomaterials initiated by an optical pump pulse, in imaging, diffraction, spectroscopy, and their combinations. It has demonstrated its capability in the studies of phase transitions, mechanical vibrations, and chemical reactions. Moreover, electrons can directly interact with photons via the near field component of light scattering by nanostructures, and either gain or lose light quanta discretely in energy. By energetically selecting those electrons that exchanged photon energies, we can map this photon-electron interaction, and the technique is termed photon-induced near field electron microscopy (PINEM). Here, we give an account of the theoretical understanding of PINEM. Experimentally, nanostructures such as a sphere, cylinder, strip, and triangle have been investigated. Theoretically, time-dependent Schrödinger and Dirac equations for an electron under light are directly solved to obtain analytical solutions. The interaction probability is expressed by the mechanical work done by an optical wave on a traveling electron, which can be evaluated analytically by the near field components of the Rayleigh scattering for small spheres and thin cylinders, and numerically by the discrete dipole approximation for other geometries. Application in visualization of plasmon fields is discussed.
\end{abstract}

Keywords: ultrafast electron microscopy, electron energy spectroscopy, nanoparticle, plasmon, near field

\section{INTRODUCTION}

Plasmons dictate the optical responses of metals in the visible range, and localized surface plasmons (LSP) in metallic nanoparticles exhibit a subwavelength confinement of electromagnetic waves and field enhancement. ${ }^{1-3}$ Therefore, LSP plays an important role in plasmonics, subdiffraction wave guides, and chemical sensing. For example, surface enhanced Raman scattering (SERS) can exhibit an enhancement of $10^{8} \sim 10^{14}$ by utilizing plasmon (resonance), making a single molecule detection possible. Plasmon excitation and field enhancement in nanoparticles are highly sensitive to the geometry of the particle, and understanding their relation to structure is of great importance in developing those fields. Therefore, experimental methods to probe the field enhancement are indispensable in advancing plasmon studies.

Nelayah et. al. invoked spectral imaging (SI) to map plasmons in a nanoprism using scanning transmission electron microscopy and electron energy loss spectroscopy (STEM-EELS), and resolved three different plasmon modes. ${ }^{4,5}$ It was also also demonstrated that energy-filtered transmission electron microscopy (EFTEM) can be equivalently employed to obtain the same information. ${ }^{6}$ Plasmons in nanoparticles of other geometries were also visualized by SI methods. ${ }^{7-21}$ Consequently, simulations of EELS of nanoparticles have been studied extensively to reveal their relation to plasmon fields. ${ }^{22-24}$ In particular, to investigatte SERS activity, Guiton et. al. employed STEM-EELS to probe the fields of plasmons in silver nanorods, and correlated this with far-field scattering spectroscopy. ${ }^{13}$ They further investigated EELS with a discrete dipole approximation (DDA) simulation, ${ }^{24}$ and compared it to the optical excitation. ${ }^{25}$ It was demonstrated that while EELS can excite plasmon modes that are not available to optical excitation, EELS can also be insensitive to interparticle junction field enhancement.

García de Abajo et. al. proposed electron energy gain spectroscopy (EEGS), ${ }^{26}$ which achieves electron energy spectroscopy with an energy resolution determined by laser bandwidth, by exciting a plasmon mode in

Further author information: (Send correspondence to A.H.Z.)

S.T.P.: E-mail: stpark@caltech.edu, Telephone: 16263956589

A.H.Z.: E-mail: zewail@caltech.edu, Telephone: 16263952611

Ultrafast Imaging and Spectroscopy, edited by Zhiwen Liu, Proc. of SPIE Vol. 8845, $884506 \cdot$ ㄷ 2013 SPIE · CCC code: 0277-786X/13/\$18 · doi: 10.1117/12.2023082 

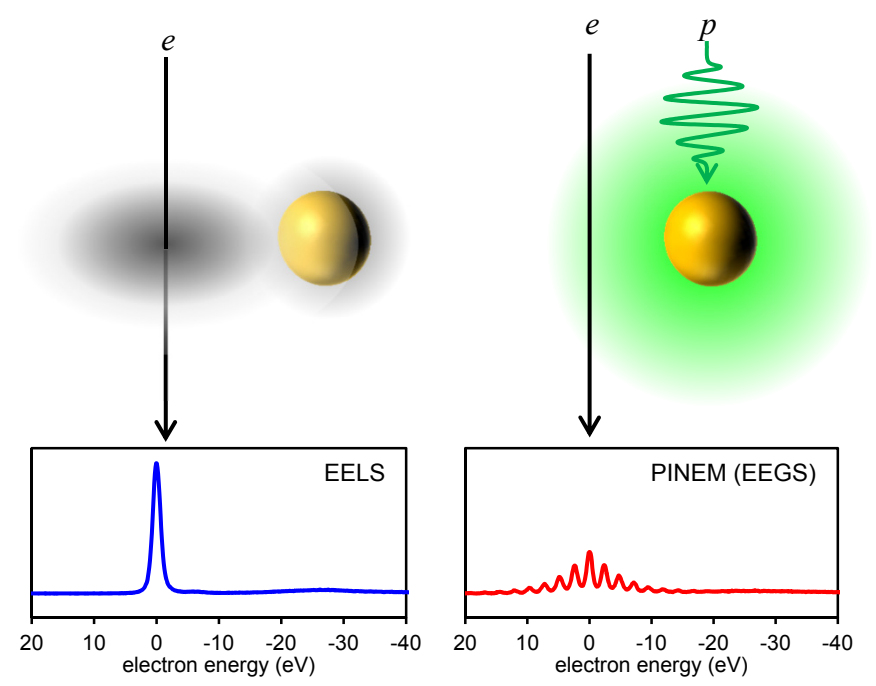

Figure 1. Comparison of interactions in EELS and PINEM.

a nanoparticle with external light and probing it with the energy gain process of a swift electron. Barwick et. al. demonstrated this technique for nanostructures with a femtosecond laser in ultrafast electron microscopy (UEM), and termed it photon-induced near field electron microscopy (PINEM), emphasizing the capability to spatially map the photon-electron interaction. ${ }^{27,28}$ Theoretical accounts have been given by various groups. ${ }^{29-31}$

Here, we present the imaging aspect of PINEM, in particular, the capability to visualize the optical near field by spatially mapping the photon-electron interaction, and compare it to that by EELS. In EELS, swift electrons induce a transient electric field with a continuous spectrum of frequency and excite plasmons in nearby particles. In PINEM, a single-frequency optical pulse drives plasmons in particles, and electrons interact with confined plasmon fields (scattering). Therefore, PINEM maps the field of a single plasmon excited by light, whereas EELS maps the excitation efficiency of plasmons. We demonstrate this for a dielectric sphere, a silver nanorod, and a silver triangle, and discuss the plasmon field distribution with respect to PINEM images.

\section{THEORETICAL}

\subsection{Electron-plasmon coupling}

In EELS (see Fig. 1), a moving electron imposes a spectrum of the electric field of

$$
\tilde{\mathbf{E}}_{e}(\mathbf{r}, \omega)=\frac{2 e \omega}{\epsilon v_{e}^{2} \gamma_{\epsilon}} \exp \left[i \frac{\omega}{v_{e}} z\right]\left[\frac{i}{\gamma_{\epsilon}} K_{0}\left(\frac{\omega}{v_{e}} \frac{b}{\gamma_{\epsilon}}\right) \hat{\mathbf{v}}_{e}-K_{1}\left(\frac{\omega}{v_{e}} \frac{b}{\gamma_{\epsilon}}\right) \hat{\mathbf{b}}\right],
$$

where $b$ is the impact parameter, $\gamma_{\epsilon}=\sqrt{1-\epsilon \frac{v_{e}^{2}}{c^{2}}}$ is the Lorentz contraction factor for an electron moving at the velocity, $v_{e}$, in dielectric medium, $\epsilon$, and $K_{n}$ is the modified bessel function of the second kind. ${ }^{22}$ This transient electric field excites plasmons in a nanoparticle nearby, and the electron loses an energy quanta of $\hbar \omega$. The plasmon field is determined by the geometry and material of the particle, and the transient field of a swift electron. Classically, the efficiency of the energy loss process is calculated by the rate of mechanical work performed on the electron by the electric field,

$$
\Gamma_{E E L S}(\omega)=\frac{e}{\pi \hbar \omega} \int d t \operatorname{Re}\left[\mathbf{v}_{e} \cdot\left\{\tilde{\mathbf{E}}\left(\mathbf{r}_{e}, \omega\right) e^{-i \omega t}\right\}\right],
$$

where $\mathbf{r}_{e}=\mathbf{v}_{e} t$ and $\tilde{\mathbf{E}}$ is the plasmon field of the particle. In EELS, an electron both excites and probes plasmons, producing an efficiency map. A strong spatial dependence in Eqn. (1) results in a significant excitation of plasmon only when the electron trajectory is very close to the plasmon mode. The angular dependence of the radial component allows excitations of the plasmon modes of an antisymmetric field when the electron impinges inside the particle, unlike light which, in the Rayleigh regime, can only excite a dipole mode. ${ }^{24}$ 


\subsection{Photon-electron coupling}

Contrastingly, in PINEM (see Fig. 1), plasmons are excited by the linearly-polarized planar wave incident light of

$$
\tilde{\mathbf{E}}_{p}(\vec{r}, t)=\tilde{E}_{0} \hat{\mathbf{x}} \exp \left[i\left(k_{p} z-\omega_{p} t\right)\right] \exp \left[-\frac{(z-c(t+\tau))^{2}}{4 c^{2} \sigma t_{p}^{2}}\right],
$$

where $\sigma t_{p}$ is the photon duration, and $\tau$ is the delay between electron and photon pulses, and electrons interact with the plasmon fields and gain and/or lose multiple light quanta, $n \hbar \omega_{p}$. A detailed derivation of the transition probability of PINEM in a non-relativistic formulation was given in Ref. 30 and summarized in Ref. 32, where the time-dependent 1D Schrödinger equation of a free electron under a scattered electromagnetic wave was analytically solved to first order. The relativistic formulation was also given in Ref. 32, and it was shown that the PINEM probability in a non-relativistic formulation is, to first order, equivalent to that in a relativistic formulation as long as the relativistic velocity of an electron is used. The temporal filter aspect of PINEM was given in Ref. 33 and utilized to characterize the temporal spread and the energy-time correlation of an electron pulse in Ref. 34.

When the total density of a propagating electron packet is given by $P_{e}\left(z^{\prime}\right)$ on a moving frame, $z^{\prime} \equiv z-v_{e} t$, the electron density in the $n$th order state becomes

$$
P_{n}\left(z^{\prime}\right)=P_{e}\left(z^{\prime}\right) Q_{n}\left(z^{\prime} ; \tau\right)
$$

and the $n$th order transition probability, $Q_{n}$, is given by

$$
\begin{aligned}
& Q_{n}\left(z^{\prime} ; \tau\right)=\left|\xi_{n}\right|^{2} \\
& \xi_{n}\left(z^{\prime} ; \tau\right)=\left(\frac{\tilde{F}_{0}}{\left|\tilde{F}_{0}\right|}\right)^{n} J_{n}\left\{-\frac{e\left|\tilde{F}_{0}\right|}{\hbar \omega_{p}} \exp \left[-\frac{\left(z^{\prime}-v_{e} \tau\right)^{2}}{4 v_{e}^{2} \sigma t_{p}^{2}}\right]\right\}
\end{aligned}
$$

where $-e$ is the electron charge, and $J_{n}$ is Bessel function of the first kind. The exponential function describes the overlapping of electrons with the incident light pulse at the position of particle, $z=0$, in a slowly-varying envelope approximation. ${ }^{30}$ For a weak interaction, we obtain $Q_{+1} \propto|\tilde{F}|^{2}$ because $J_{n}(\Omega) \approx \frac{1}{n !}\left(\frac{\Omega}{2}\right)^{n}$ for a small argument. The field integral, $\tilde{F}$, is defined as

$$
\tilde{F}(x, y) \equiv v_{e} \int_{-\infty}^{+\infty} d t^{\prime \prime} \tilde{E}_{z}\left(x, y, v_{e} t^{\prime \prime}, t^{\prime \prime}\right)
$$

which is mechanical work (per charge) performed by an electromagnetic wave (of $\omega_{p}$ ) on a traveling electron at $z^{\prime \prime} \equiv v_{e} t^{\prime \prime}$, and therefore equivalent to Eqn. (2). By factoring out the time dependence, $e^{-i \omega_{p} t}$, Eqn. (6) is reduced to

$$
\tilde{F}_{0}(x, y)=\int_{-\infty}^{+\infty} d z^{\prime \prime} \tilde{E}_{z}\left(x, y, z^{\prime \prime}, 0\right) \exp \left[-i\left(\frac{\omega_{p}}{v_{e}}\right) z^{\prime \prime}\right]
$$

which becomes the Fourier transform of the $z$ component of the scattered electric field at a spatial frequency $\Delta k \equiv \frac{\omega_{p}}{v_{0}}$.

The field integral is determined by the scattered light and the electron velocity, and it describes the magnitude of photon-electron interaction. Therefore, the task of PINEM image simulation reduces to calculating the light scattering of a nanoparticle and then evaluating Eqn. (7) as a function of position, $(x, y)$. Note that the scattered electric field is a three-component vector quantity in $3 \mathrm{D}(x, y, z)$ space, while the field integral is a scalar quantity of 1D $(z)$ projectile trajectories, in 2D $(x, y)$ subspace. Similarly, an object is a 3D distribution of density, $\rho(x, y, z)$, while a TEM image, $I(x, y)$, is a set of projections (integrations of electron scattering density along $z$ ) in the $x-y$ plane. 


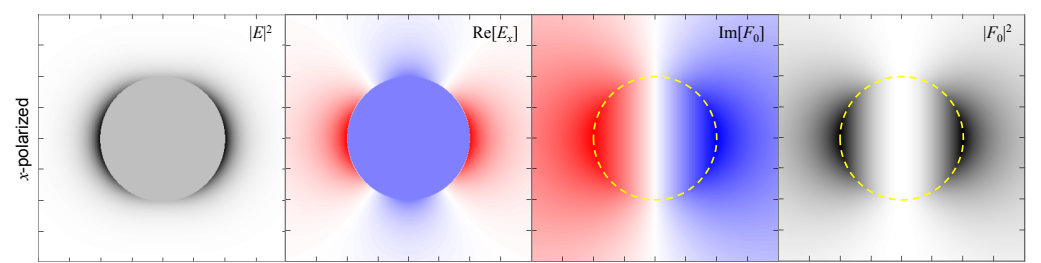

Figure 2. Scattered electric fields $(\vec{E})$, field integral $\left(\tilde{F}_{0}\right)$, and first-order transition probability $\left(\left|\tilde{F}_{0}\right|^{2}\right)$, for a dielectric sphere at $519 \mathrm{~nm}$ excitation.

\section{RESULT}

Light scattering by nanoparticles can be analytically expressed by Mie theory ${ }^{35,36}$ for spheres or cylinders of arbitrary sizes, and by Rayleigh dipole approximation ${ }^{37,38}$ for small ellipsoids. Mie theory has been extended to multilayer spheres ${ }^{39}$ and spheres with inhomogeneous refractive indices. ${ }^{40}$ For other geometries, numerical methods such as finite difference time domain (FDTD) approach,${ }^{41}$ discrete dipole approximation (DDA), ${ }^{42}$ or boundary element method (BEM) ${ }^{22,43}$ can be used. Then, Eqn. (7) can be numerically evaluated with the scattered electric field calculated by the methods mentioned above.

Analytical formulations of plasmon excitation by a swift electron have been studied for various geometries (see Ref. 22 and references therein). DDA and BEM can be easily modified to implement numerical simulations of such excitations for arbitrary geometries by replacing the incident light with Eqn. (1) for each electron trajectory.

\subsection{Sphere}

Near field approximation ${ }^{30}$ of Rayleigh scattering for $r>a$ is given by

$$
\begin{aligned}
E_{x} & \approx \tilde{E}_{0} \chi_{s} a^{3} \frac{3 x^{2}-r^{2}}{3 r^{5}} e^{-i \omega_{p} t} \\
E_{y} & \approx \tilde{E}_{0} \chi_{s} a^{3} \frac{x y}{r^{5}} e^{-i \omega_{p} t} \\
E_{z} & \approx \tilde{E}_{0} \chi_{s} a^{3} \frac{z x}{r^{5}} e^{-i \omega_{p} t},
\end{aligned}
$$

where $a$ is the radius and $\chi_{s} \equiv \frac{3}{\epsilon+2}(\epsilon-1)$. It allows one to obtain an analytical expression for Eqn. (7) for $b>a$ as

$$
\tilde{F}_{0}=-i \tilde{E}_{0} \cos \phi \chi_{s} \frac{2}{3} a^{3} \Delta k^{2} K_{1}[\Delta k b],
$$

where $b=\sqrt{x^{2}+y^{2}}$ and $\cos \phi=\frac{x}{b}$. Fig. 2 (a) and (b) plot the scattered intensity and the electric field $x$ component using Eqn. (8) on the $x-y$ plane at $z=0$. On the plane of $z=0, E_{z}$ vanishes due to its symmetry, and $E_{x}$, which is parallel to the incident light polarization, is a major component. Along the $x$ axis at $y=0$, $E_{x}$ attenuates with $r^{-3}$, resulting in the decay length of $\delta_{E}=\left\{\lim _{b \rightarrow a} \frac{\partial \log |E|}{\partial b}\right\}^{-1}=a / 3$, and the intensity, $I \propto|E|^{2}$, has a decay length of $a / 6$. Fig. 2 (c) and (d) plot the field integral and the first order transition probability using Eqns. (9) and (5), respectively, in the $x-y$ plane. Eqn. (9) decays approximately reciprocally for $b>a$ due to the bessel function, such that its decay length becomes $\delta_{F}=\left\{\lim _{b \rightarrow a} \frac{\partial \log \left|\tilde{F}_{0}\right|}{\partial b}\right\}^{-1}=a$. Eqn. (7) is numerically evaluated for $b<a$, and it shows that the field integral monotonically decreases as $b \rightarrow 0$ and vanishes at $b=0$. The polarization angle dependence is given by $\cos \phi$ in Eqn. (9), and results in a node at $x=0$. Since $\tilde{F}_{0}$ decays with $a, Q_{+1} \propto\left|\tilde{F}_{0}\right|^{2}$ decays with $a / 2$.

Outside the particle, $|\vec{E}|^{2}$ on the $x-y$ plane $(z=0)$ and $\left|\tilde{F}_{0}\right|^{2}$ are generally correlated, even though the phase of $\tilde{F}_{0}$ does not match that of $E_{x}$ or $E_{y}$. One may choose to compare to $E_{z}$ on an elevated $x-y$ plane $(z=2 a$ where $E_{z}$ shows optima), in which case the angular dependence matches but the radial dependence still deviates. As stated before, we cannot exactly compare a $3 \mathrm{D}$ vector quantity $(\vec{E})$ and a $2 \mathrm{D}$ scalar quantity $\left(\tilde{F}_{0}\right)$, since $\tilde{F}_{0}$ is a (Fourier component of $z$ component) subset of $\vec{E}(\mathbf{k})$. 


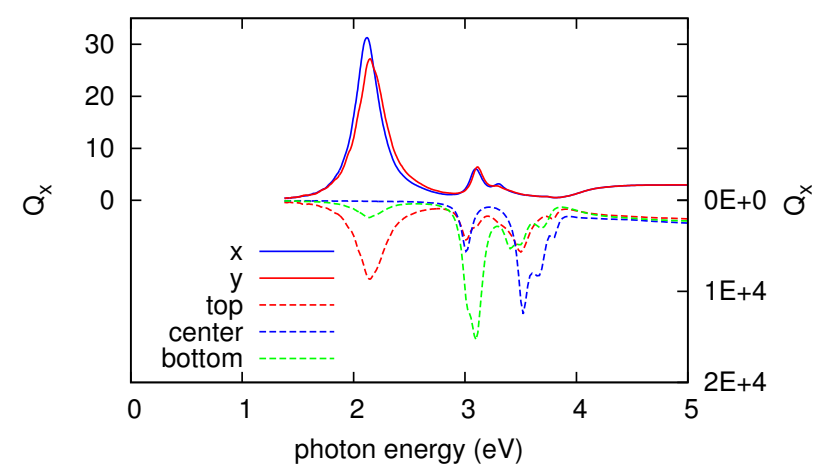

Figure 3. Optical and electric excitation efficiencies of a silver nanotriangle.

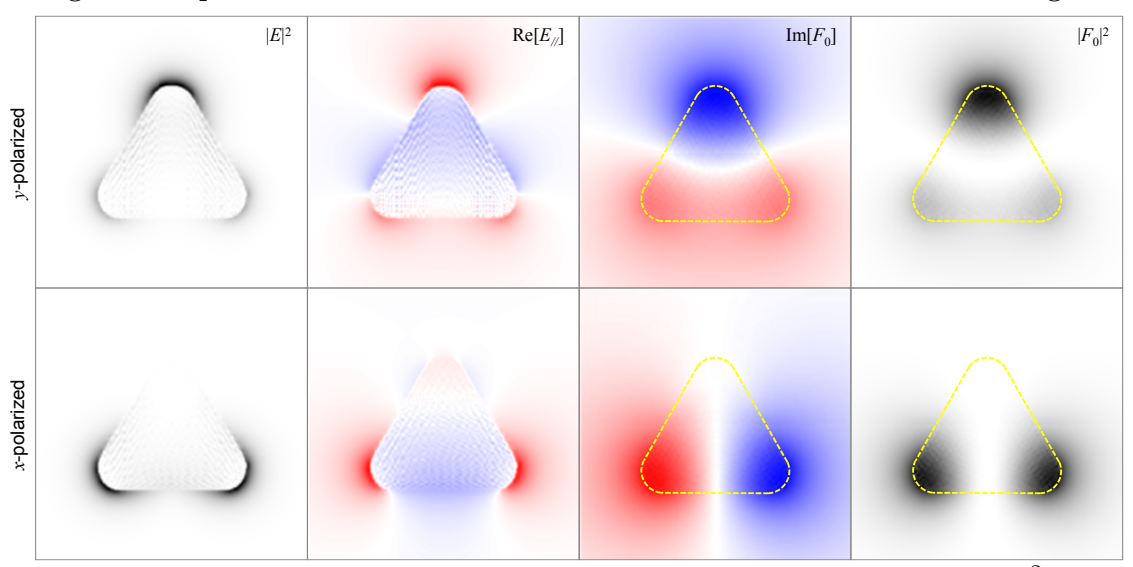

Figure 4. Scattered electric fields $(\vec{E})$, field integrals $\left(\tilde{F}_{0}\right)$, and transition probabilities $\left(\left|\tilde{F}_{0}\right|^{2}\right)$ for a silver nanotriangle at $\hat{\mathbf{y}}$ and $\hat{\mathbf{x}}$ polarized $519 \mathrm{~nm}$ excitations. Note that electric fields were Gaussian-filtered $(\sigma=1$ pixel $)$ to reduce DDA artifact.

\subsection{Triangle}

A silver nanotriangle of $130 \mathrm{~nm}$ width and $20 \mathrm{~nm}$ height (see Ref. 44 for the geometry) was studied using DDA simulations (with $2 \mathrm{~nm}$ grid size) for optical excitations. (Readers are referred to Refs. 4, 6, 5, 7, 23, and 17 for electric excitation.) Vertices and sides were rounded with a radius of curvature of $10 \mathrm{~nm}$ to mimic the particle studied previously. ${ }^{44}$ Fig. 3 plots extinction efficiencies for planar wave optical excitations and for swift electron transient field excitations at top, center, and bottom of the nanotriangle. The positions of resonance peaks generally coincide, but the intensities are different. For electric excitations, the efficiency spectra strongly depend on the position of impinging electrons, as demonstrated in SI measurements. ${ }^{4,5}$ For planar wave excitations, polarization dependence is very small due to the three fold symmetry of the particle.

Fig. 4 shows electric fields, field integral, and transition probability at $519 \mathrm{~nm}$ excitations. They are similar to those of a sphere in Fig. 2, except fields are concentrated around vertices. Namely, it can be regarded as a dipole mode with two vertices, or with one sharp vertex and one dull edge. Similarly, PINEM images are generally correlated to scattering intensities.

Figs. 5 and 6 show scattering intensities and PINEM images at various resonance energies, respectively. $582 \mathrm{~nm}$ resonance is dominated by a dipole excitation. $399 \mathrm{~nm}$ excitation exhibits a quadrupole characteristic. PINEM is stronger at edges than vertices at higher photon energies. $250 \mathrm{~nm}$ excitation seems to be a dipole mode. Similar to $519 \mathrm{~nm}$ excitation, PINEM images are generally correlated to scattering intensities at these resonance energies.

STEM-EELS and EFTEM images ${ }^{4,5}$ always show a three-fold symmetry for a nanotriangle, because they map electric excitation efficiency and the polarization of electric transient field cannot be controlled. Polarization 


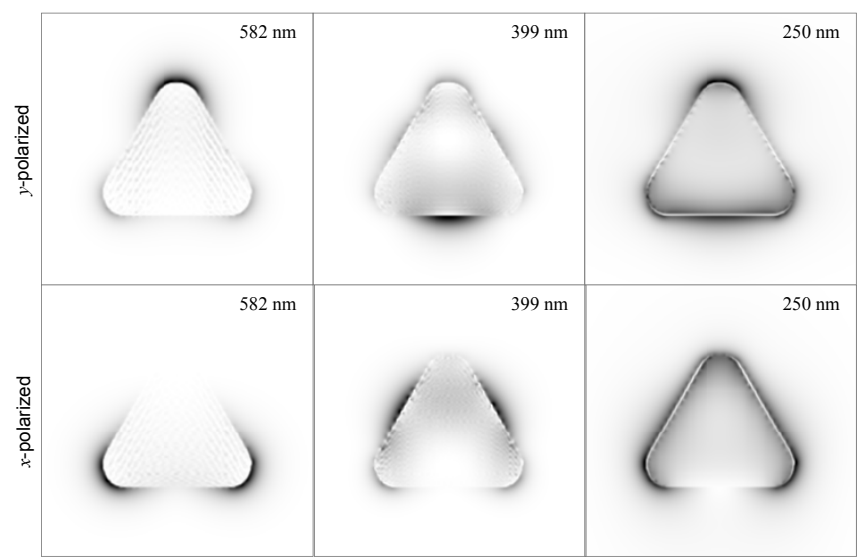

Figure 5. Scattering intensities of a silver nanotriangle at 582, 399, and $250 \mathrm{~nm}$. Note that electric fields were Gaussianfiltered $(\sigma=1$ pixel) to reduce DDA artifact.

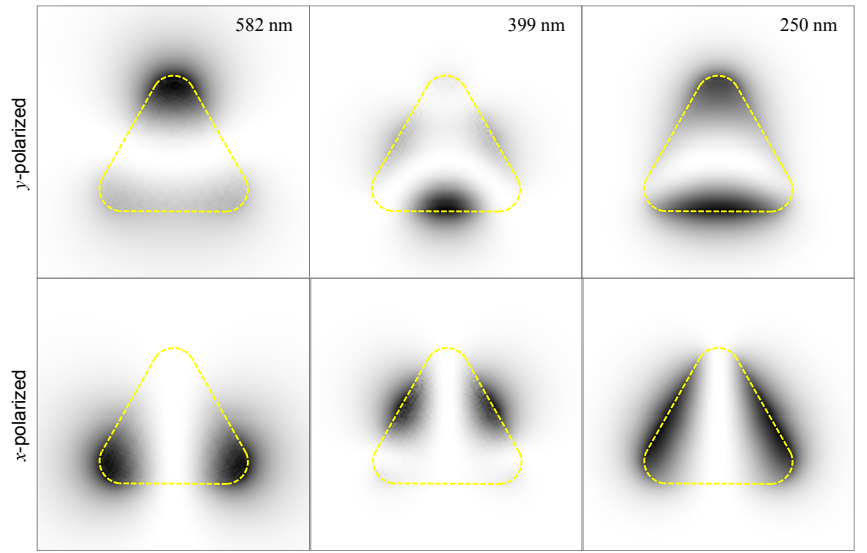

Figure 6. Transition probabilities of a silver nanotriangle at 582, 399, and $250 \mathrm{~nm}$.

dependence of PINEM images reveals more information, and PINEM images are evidently correlated to plasmon modes by optical excitations.

\subsection{Rod}

A silver nanorod of $192 \mathrm{~nm}$ length and $20 \mathrm{~nm}$ diameter (see Ref. 13 for the geometry) was studied using DDA simulations (with $1 \mathrm{~nm}$ grid size) for optical excitations. (Readers are referred to Refs. 13, 24, and 25 for electric excitation.) Ends were rounded with a radius of curvature of $10 \mathrm{~nm}$. Fig. 7 plots extinction efficiencies for planar wave optical excitation and for swift electron transient field excitations at end, center, and side of the nanorod. Note that DDA calculations at photon energies below $1.5 \mathrm{eV}$ (longer wavelength) were not carried out here due to slow convergences. The resonance peaks of two different excitations coincide at 2.5, 3.1, and $3.6 \mathrm{eV}$ (which correspond to $l=3, l=5$, and $l^{\prime}=1$, respectively, where $l=1$ denotes a dipole mode which shows one node in electric fields), but the electric excitation shows stronger peaks at 2.0, 2.9, and $3.2 \mathrm{eV} .{ }^{24}$ Electric excitation also shows the position dependence of the efficiency spectra, resulting from the plasmon mode distribution. ${ }^{13}$ Planar wave excitation exhibits a strong anisotropy due to the geometry (large aspect ratio) of the particle such that the polarization dependence is complementary. The polarization parallel to the axis shows resonances at lower photon energies (longer wavelengths) compared to the perpendicular polarization which only shows a broad resonance at $\sim 3.6 \mathrm{eV}$.

Fig. 8 shows electric fields, field integral, and transition probability at $519 \mathrm{~nm}$ excitations. Perpendicular excitation shows the same dipole excitation behavior as an infinite thin cylinder, except at truncated ends. 20 $\mathrm{nm}$ radius of the rod is small enough to fulfill Rayleigh approximation criteria and $192 \mathrm{~nm}$ length is long enough to treat the middle region as an infinite cylinder. However, parallel excitation shows a multipole behavior $(l=3)$ 


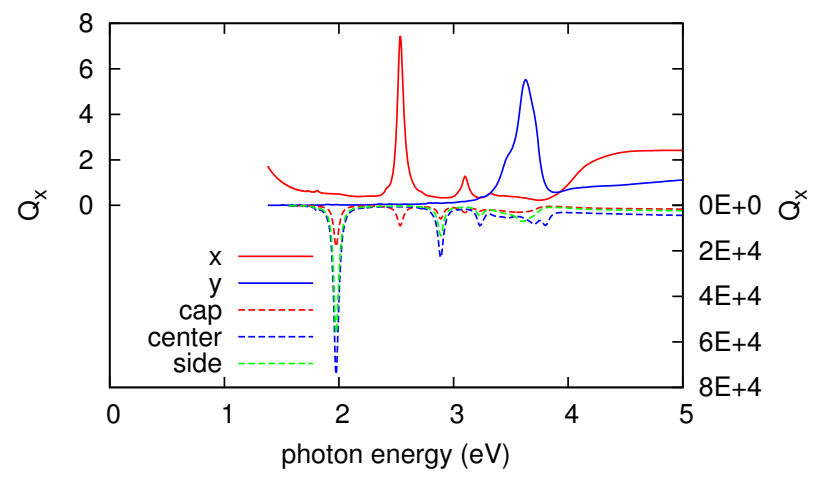

Figure 7. Optical and electric excitation efficiencies of a silver nanorod.

\begin{tabular}{|c|c|c|c|c|}
\hline$x$ & $\Longleftrightarrow$ & $\Longrightarrow$ & $\operatorname{Im}\left[F_{0}\right]$ & ${ }_{-\infty}$ \\
\hline$y$ & $(\sqrt{-}-1)$ & coin 10 & 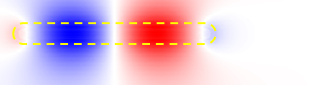 & $m+\cdots$ \\
\hline
\end{tabular}

Figure 8. Scattered electric fields $(\vec{E})$, field integral $\left(\tilde{F}_{0}\right)$, and transition probabilities $\left(\left|\tilde{F}_{0}\right|^{2}\right)$ for a silver nanorod at $\hat{\mathbf{y}}$ and $\hat{\mathbf{x}}$ polarized $519 \mathrm{~nm}$ excitations. Note that electric fields were Gaussian-filtered $(\sigma=1$ pixel $)$ to reduce DDA artifact.

with much stronger intensities in the middle. Furthermore, $|\tilde{E}|$ is dominated by $\tilde{E}_{x}$ at ends, but by $\tilde{E}_{y}$ in the middle, which results in different numbers of nodes between $|\tilde{E}|^{2}$ and $\operatorname{Re}\left[\tilde{E}_{x}\right]$, and the PINEM image is correlated to $|\vec{E}|^{2}$.

Figs. 9 and 10 show scattering intensities and PINEM images at various resonance energies, respectively. Good correlations between scattering intensities and PINEM images are readily seen. Perpendicular excitations all show PINEM images similar to that at $519 \mathrm{~nm}$, which is a dipole excitation in a cylinder. Parallel excitations show a strong wavelength dependence. In particular, $400 \mathrm{~nm}$ shows (linear) hexapole $(l=5)$ behavior, and the PINEM image shows six maxima along the rod axis. $342 \mathrm{~nm}$ corresponds to $3.6 \mathrm{eV}$ peak in Fig. 7 , which shows a strong excitation for perpendicular polarization and almost no excitation for parallel polarization. Consequently, the PINEM image for $342 \mathrm{~nm}$ parallel polarization shows non-resonant behavior, namely a weak dipole excitation.

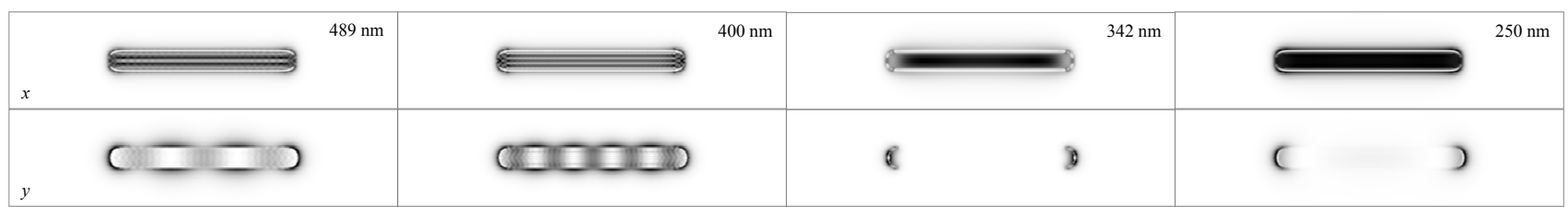

Figure 9. Scattering intensities of a silver nanorod at 489, 400, 342, and $250 \mathrm{~nm}$. Note that electric fields were Gaussianfiltered $(\sigma=1$ pixel) to reduce DDA artifact.

\begin{tabular}{|c|c|c|c|c|c|}
\hline$x$ & nan & $400 \mathrm{~nm}$ & $342 \mathrm{~nm}$ & 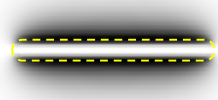 & $250 \mathrm{~nm}$ \\
\hline$y$ & 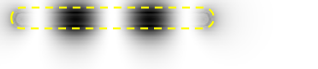 & 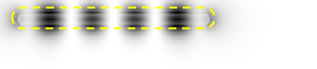 & \& & $\varepsilon$ & \\
\hline
\end{tabular}

Figure 10. Transition probabilities of a silver nanorod at 489, 400, 342, and $250 \mathrm{~nm}$. 


\section{SUMMARY}

Although EELS and PINEM share an equivalent probing mechanism, which is mechanical work performed by an electromagnetic field on a moving electron, their excitation mechanisms are different. The optical excitation mechanism of PINEM enables one to visualize the plasmon field in optical excitation, while EELS only produces an efficiency map of electric excitation.

A silver nanorod and a silver triangle are two examples whose plasmon excitations are well studied with EELS techniques. The spatial profiles of PINEM for those geometries were investigated in relation to the plasmon scattered field. In general, it was observed that PINEM images are correlated to scattering intensities outside of particles, such that PINEM intensity is strong where the scattering intensity is strong, and it allows one to identify optical hot spots at given light polarizations.

\section{ACKNOWLEDGMENTS}

This work was supported by the National Science Foundation and the Air Force Office of Scientific Research in the Center for Physical Biology funded by the Gordon and Betty Moore Foundation.

\section{REFERENCES}

[1] Maier, S. A., [Plasmonics: Fundamentals and Applications], Springer, New York (2007).

[2] Pitarke, J. M., Silkin, V. M., Chulkov, E. V., and Echenique, P. M., "Theory of surface plasmons and surface-plasmon polaritons," Rep. Prog. Phys. 70(1), 1-87 (2007).

[3] Zhang, J., Zhang, L., and Xu, W., "Surface plasmon polaritons: physics and applications," J. Phys. D: Appl. Phys. 45(11), 113001 (2012).

[4] Nelayah, J., Kociak, M., Stéphan, O., García de Abajo, F. J., Tencé, M., Henrard, L., Taverna, D., PastorizaSantos, I., Liz-Marzán, L. M., and Colliex, C., "Mapping surface plasmons on a single metallic nanoparticle," Nat. Phys. 3(5), 348-353 (2007).

[5] Nelayah, J., Kociak, M., Stephan, O., Geuquet, N., Henrard, L., García de Abajo, F. J., Pastoriza-Santos, I., Liz-Marzan, L. M., and Colliex, C., "Two-dimensional quasistatic stationary short range surface plasmons in flat nanoprisms," Nano Lett. 10(3), 902-907 (2010).

[6] Nelayah, J., Gu, I., Sigle, W., Koch, C. T., Pastoriza-Santos, I., Liz-Marzán, L. M., and van Aken, P. A., "Direct imaging of surface plasmon resonances on single triangular silver nanoprisms at optical wavelength using low-loss EFTEM imaging," Opt. Lett. 34(7), 1003-1005 (2009).

[7] Rang, M., Jones, A. C., Zhou, F., Li, Z. Y., Wiley, B. J., Xia, Y. N., and Raschke, M. B., "Optical near-field mapping of plasmonic nanoprisms," Nano Lett. 8(10), 3357-3363 (2008).

[8] Schaffer, B., Hohenester, U., Trugler, A., and Hofer, F., "High-resolution surface plasmon imaging of gold nanoparticles by energy-filtered transmission electron microscopy," Phys. Rev. B 79(4), 041401 (2009).

[9] Sigle, W., Nelayah, J., Koch, C. T., and van Aken, P. A., "Electron energy losses in Ag nanoholes-from localized surface plasmon resonances to rings of fire," Opt. Lett. 34(14), 2150-2152 (2009).

[10] Arslan, I., Hyun, J. K., Erni, R., Fairchild, M. N., Hersee, S. D., and Muller, D. A., "Using electrons as a high-resolution probe of optical modes in individual nanowires," Nano Lett. 9(12), 4073-4077 (2009).

[11] Hohenester, U., Ditlbacher, H., and Krenn, J. R., "Electron-energy-loss spectra of plasmonic nanoparticles," Phys. Rev. Lett. 103(10), 106801 (2009).

[12] Koh, A. L., Bao, K., Khan, I., Smith, W. E., Kothleitner, G., Nordlander, P., Maier, S. A., and McComb, D. W., "Electron energy-loss spectroscopy (EELS) of surface plasmons in single silver nanoparticles and dimers: Influence of beam damage and mapping of dark modes," ACS Nano 3(10), 3015-3022 (2009).

[13] Guiton, B. S., Iberi, V., Li, S. Z., Leonard, D. N., Parish, C. M., Kotula, P. G., Varela, M., Schatz, G. C., Pennycook, S. J., and Camden, J. P., "Correlated optical measurements and plasmon mapping of silver nanorods," Nano Lett. 11(8), 3482-3488 (2011).

[14] Alber, I., Sigle, W., Muller, S., Neumann, R., Picht, O., Rauber, M., van Aken, P. A., and Toimil-Molares, M. E., "Visualization of multipolar longitudinal and transversal surface plasmon modes in nanowire dimers," ACS Nano 5(12), 9845-9853 (2011). 
[15] Henry, A. I., Bingham, J. M., Ringe, E., Marks, L. D., Schatz, G. C., and Van Duyne, R. P., "Correlated structure and optical property studies of plasmonic nanoparticles," J. Phys. Chem. C 115(19), 9291-9305 (2011).

[16] Andersen, K., Jacobsen, K. W., and Thygesen, K. S., "Spatially resolved quantum plasmon modes in metallic nano-films from first-principles," Phys. Rev. B 86(24), 245129 (2012).

[17] Blaber, M. G., Henry, A. I., Bingham, J. M., Schatz, G. C., and Van Duyne, R. P., "LSPR imaging of silver triangular nanoprisms: Correlating scattering with structure using electrodynamics for plasmon lifetime analysis," J.Phys. Chem. C 116(1), 393-403 (2012).

[18] Duan, H. G., Fernandez-Dominguez, A. I., Bosman, M., Maier, S. A., and Yang, J. K. W., "Nanoplasmonics: Classical down to the nanometer scale," Nano Lett. 12(3), 1683-1689 (2012).

[19] Edwards, P. R., Jagadamma, L. K., Bruckbauer, J., Liu, C. W., Shields, P., Allsopp, D., Wang, T., and Martin, R. W., "High-resolution cathodoluminescence hyperspectral imaging of nitride nanostructures," Microsc. Microanal. 18(6), 1212-1219 (2012).

[20] Mazzucco, S., Geuquet, N., Ye, J., Stephan, O., Van Roy, W., Van Dorpe, P., Henrard, L., and Kociak, M., "Ultralocal modification of surface plasmons properties in silver nanocubes," Nano Lett. 12(3), 1288-1294 (2012).

[21] Myroshnychenko, V., Nelayah, J., Adamo, G., Geuquet, N., Rodriguez-Fernandez, J., Pastoriza-Santos, I., MacDonald, K. F., Henrard, L., Liz-Marzan, L. M., Zheludev, N. I., Kociak, M., and García de Abajo, F. J., "Plasmon spectroscopy and imaging of individual gold nanodecahedra: A combined optical microscopy, cathodoluminescence, and electron energy-loss spectroscopy study," Nano Lett. 12(8), 4172-4180 (2012).

[22] García de Abajo, F. J., "Optical excitations in electron microscopy," Rev. Mod. Phys. 82(1), 209-275 (2010).

[23] Geuquet, N. and Henrard, L., "EELS and optical response of a noble metal nanoparticle in the frame of a discrete dipole approximation," Ultramicroscopy 110(8), 1075-1080 (2010).

[24] Bigelow, N. W., Vaschillo, A., Iberi, V., Camden, J. P., and Masiello, D. J., "Characterization of the electron- and photon-driven plasmonic excitations of metal nanorods," ACS Nano 6(8), 7497-7504 (2012).

[25] Mirsaleh-Kohan, N., Iberi, V., Simmons, P. D., Bigelow, N. W., Vaschillo, A., Rowland, M. M., Best, M. D., Pennycook, S. J., Masiello, D. J., Guiton, B. S., and Camden, J. P., "Single-molecule surface-enhanced raman scattering: Can STEM/EELS image electromagnetic hot spots?," J. Phys. Chem. Lett. 3(16), 2303-2309 (2012).

[26] García de Abajo, F. J. and Kociak, M., "Electron energy-gain spectroscopy," New J. Phys. 10, 073035 (2008).

[27] Barwick, B., Flannigan, D. J., and Zewail, A. H., "Photon-induced near-field electron microscopy," Nature 462(7275), 902-906 (2009).

[28] Flannigan, D. J., Barwick, B., and Zewail, A. H., "Biological imaging with 4D ultrafast electron microscopy," Proc. Natl. Acad. Sci. USA 107(22), 9933-9937 (2010).

[29] García de Abajo, F. J., Asenjo-Garcia, A., and Kocíak, M., "Multiphoton absorption and emission by interaction of swift electrons with evanescent light fields," Nano Lett. 10(5), 1859-1863 (2010).

[30] Park, S. T., Lin, M., and Zewail, A. H., "Photon-induced near-field electron microscopy (PINEM): Theoretical and experimental," New J. Phys. 12, 123028 (2010).

[31] Howie, A., "Photon interactions for electron microscopy applications," Eur. Phys. J.-Appl. Phys. 54(3), 33502 (2011).

[32] Park, S. T. and Zewail, A. H., "Relativistic effects in photon-induced near-field electron microscopy," $J$. Phys. Chem. A 116(46), 11128-11133 (2012).

[33] Park, S. T. and Zewail, A. H., "Enhancing image contrast and slicing electron pulses in 4D near field electron microscopy," Chem. Phys. Lett. 521, 1-6 (2012).

[34] Park, S. T., Kwon, O.-H., and Zewail, A. H., "Chirped imaging pulses in four-dimensional electron microscopy: femtosecond pulsed hole burning," New J. Phys. 14, 053046 (2012).

[35] Mie, G., "Beiträge zur optik trüber medien, speziell kolloidaler metallösungen," Ann. Phys., Berlin 25, 377-445 (1908).

[36] van der Hulst, H. C., [Light Scattering by Small Particles], Wiley, New York (1957).

[37] Jackson, J. D., [Classical Electrodynamics], Wiley, New York (1975). 
[38] Gans, R., "Über die form ultramikroskopischer goldteilchen," Ann. Phys. (Lepzig) 37, 881-900 (1912).

[39] Johnson, B. R., "Light scattering by a multilayer sphere," Appl. Opt. 35(18), 3286-3296 (1996).

[40] Perelman, A. Y., "Scattering by particles with radially variable refractive indices," Appl. Opt. 35(27), 5452-5460 (1996).

[41] Courant, R., Friedrichs, K. O., and Lewy, H., "Über die partiellen differenzengleichungen der mathematischen physik," Math. Ann. 100(1), 32-74 (1928).

[42] Draine, B. T., "The discrete-dipole approximation and its application to interstellar graphite grains," Astrophys. J. 333(2), 848-872 (1988).

[43] García de Abajo, F. J. and Howie, A., "Retarded field calculation of electron energy loss in inhomogeneous dielectrics," Phys. Rev. B 65(11), 115418 (2002).

[44] Yurtserver, A., van der Veen, R. M., and Zewail, A. H., "Subparticle ultrafast spectrum imaging in 4D electron microscopy," Science 335(6064), 59-64 (2012). 